\title{
Prevención de Movimientos Repetitivos y Tenosinovitis de Quervain en el Área de Estadística del Hospital General Puyo.
}

\author{
Prevention of Repetitive Movements and Quervain's Tenosynovitis in the \\ statistics Area of the Puyo General Hospital.
}

\begin{abstract}
Esthela del Rocío Freire Ramos. ${ }^{1}$, Manolo Alexander Córdova Suarez. ${ }^{2}$, Edison Patricio Villacres Cevallos. ${ }^{3} \&$ María Fernanda Cuenca Cajamarca. ${ }^{4}$
\end{abstract}

\section{DOI: https://doi.org/10.33262/anatomiadigital.v4i3.1.1851}

\begin{abstract}
.
Introduction. The pandemic forces jobs to increase the number of hours when using new computer technologies, this causes repetitive movements and problems related to tenosynovitis in workers who carry out these activities. Objective. Implement an ergonomic prevention plan to reduce the prevalence of musculoskeletal discomfort and Quervain's tenosynovitis in the statistics
\end{abstract}

\section{Resumen.}

Introducción. La pandemia obliga a que los puestos de trabajo aumenten el número de horas al utilizar nuevas tecnologías informáticas, esto ocasiona movimientos repetitivos y problemas relacionados con tenosinovitis en los trabajadores que ejecutan estas actividades. Objetivo. Implementar un plan de prevención ergonómico para disminuir la prevalencia de molestias

\footnotetext{
${ }^{1}$ Universidad Regional Autónoma de los Andes, Posgradista. Ambato-Ecuador. Médico General. Hospital General Puyo. Puyo-Ecuador. esthe_01ef@hotmail.com (D) https://orcid.org/0000-0003-0891-3747

2 Universidad Nacional de Chimborazo, Facultad de Ingeniería, Riobamba-Ecuador, manolo.cordova@unach.edu.ec (D) https://orcid.org/0000-0001-6786-7926

3 Universidad Nacional de Chimborazo, Facultad de Ingeniería, Riobamba-Ecuador, pvillacres@ unach.edu.ec https://orcid.org/0000-0001-9518-1278

${ }^{4}$ Universidad Regional Autónoma de los Andes, Posgradista. Ambato-Ecuador. mafer1600@ hotmail.com (D) https://orcid.org/0000-0002-7353-0227
} 
area of the Puyo General Hospital.

Methods. For the diagnosis of musculoskeletal discomfort, the Nordic questionnaire was used, then an analysis was carried out prior to the execution of an ergonomic prevention program and subsequent evaluation with the OCRA Check List Method to determine the effectiveness of the measures. Results. From the application of the Nordic Questionnaire, it was observed that $65 \%$ of the workers presented musculoskeletal discomfort and $85 \%$ of the causes indicated that they were due to repetitive movements of the hand and wrist with an age range of 35 - 45 years. After applying the ergonomic prevention plan that considered the following activities: a) active pauses, b) correct location of computer peripherals, c) cell phone suspension in the recovery period, an $80 \%$ reduction in the Index score was achieved. OCRA with values of 5.25 for the right side and an acceptable risk, in addition a value of 4.87 and an optimal ergonomic risk for the left side. Conclusion: When executing an ergonomic prevention plan, the level of ergonomic risk was lowered by $80 \%$ with the application of active breaks, reduction of the time of use and improvement in the use of electronic peripherals and restricting the mobile phone during the recovery time.

Key words: Quervain's tenosynovitis, musculoskeletal, repetitive movements, Nordic Questionnaire, OCRA method. osteomusculares y tenosinovitis de Quervain en el área de estadística del Hospital General Puyo. Métodos. Para el diagnóstico de las molestias osteomusculares se utilizó el cuestionario Nórdico, luego se realizó un análisis previo a la ejecución de un programa de prevención ergonómico y evaluación posterior con el Método Check List OCRA para determinar la efectividad de las medidas. Resultados. De la aplicación del Cuestionario Nórdico se observó que un $65 \%$ de los trabajadores presentaron molestias osteomusculares y el $85 \%$ de las causas indicaron que eran debido a movimientos repetitivos de la mano y muñeca con un rango de edad 35 - 45 años. Luego de la aplicación del plan de prevención ergonómico que consideró las siguientes actividades: a) pausas activas, b) ubicación correcta de periféricos informáticos, c) suspensión de celular en el periodo de recuperación, se logró disminuir un $80 \%$ de la puntuación del Índice OCRA con valores de 5,25 para el lado derecho y un riesgo aceptable, además un valor de 4,87 y un riesgo ergonómico óptimo para el lado izquierdo. Conclusión: Al ejecutar un plan de prevención ergonómico se bajó un $80 \%$ el nivel de riesgo ergonómico con la aplicación de pausas activas, reducción del tiempo de uso y mejora en la utilización de periféricos electrónicos y restringiendo el teléfono móvil durante el tiempo de recuperación.

Palabras clave: Tenosinovitis de Quervain, osteomusculares, movimientos repetitivos, Cuestionario Nórdico, Método OCRA. 


\section{Introducción.}

Los cambios económicos y sociales provocados por el confinamiento han obligado a los hospitales a adoptar nuevas formas de trabajo, de manera repentina, y adaptaciones de la organización de las tareas por el incremento de pacientes, aumentando el uso de tecnologías de la información y comunicación (TIC'S) sin seguimiento ni verificación de las acciones del personal al realizar sus actividades (Chacín, González, \& Peñaloza, 2020). En los hospitales las áreas como la de estadística incrementaron el uso de las TIC'S por el aumento de pacientes por el COVID-19 aumentando la exposición a movimientos repetitivos desencadenantes de lesiones osteomusculares y tenosinovitis que producen cambios físicos, funcionales de las articulaciones y tejidos, generando inflamaciones, dolores leves relacionados con perdida y limitación de la fuerza muscular de los ingenieros en estadística (Moller, 2020).

En este momento los hospitales públicos en especial las áreas que manejan las TIC'S utiliza el mouse y teclado como herramientas de trabajo primarias, la exposición de largas horas a estas herramientas se asocia con un aumento de lesiones debido a trastornos musculoesqueléticos (López García \& López Mayorga, 2013). El 85\% de los trabajadores que se dedican a actividades relacionadas en el campo de la estadística presentan afecciones de extremidades superiores, principalmente las mujeres muestran un $76 \%$ de lesiones musculoesqueléticas, en una proporción de 5:1 sobre los hombres y padecen trastornos de miembros superiores (Salas Guerra \& Díaz López, 2017).

Las lesiones en el trabajo no son accidentales, sino causales y deben determinarse para crear un entorno de trabajo seguro (Cisneros-Prieto \& Cisneros-Rodríguez, 2015). Trabajar en el área de estadística implica manipular elementos periféricos e incluye ocurrencias abruptas y cambios repetitivos en los movimientos de las manos que son esenciales para realizar operaciones de apertura, toma de información y archivo de registros médicos (Espinoza Huaman, 2018). Esta actividad es muy peligrosa para la salud de los trabajadores, ya que hay poco tiempo de recuperación cuando se trabaja en una posición fija o movimientos repetitivos debido a factores como el fallo osteomuscular (Angamarca Curipoma \& Changoluisa Changoluisa, 2020).

Una herramienta importante para la investigación ergonómica en la detección y análisis de síntomas osteomusculares es el Cuestionario Nórdico de Kuorinka, este permite identificar los primeros síntomas y estimar su gravedad para una intervención temprana (Rengifo Villavicencio, 2020).

Existen muchos métodos para evaluar la ergonomía de las extremidades superiores, cada uno con sus propias características, singularidad y limitaciones, identificando aspectos relevantes como postura, movilidad y repetición de acciones realizadas por las extremidades superiores (Uribe, 2019). Una de las mejores opciones utilizadas para evaluar movimientos repetitivos y lograr resultados cualitativos y cuantitativos es el Método Check List OCRA. Este le permite evaluar el riesgo asociado con las tareas repetitivas (Sagbay, 2021). 
La misión del área de estadística es gestionar la recolección, revisión, análisis y selección de la información recogida diariamente por los servicios de urgencias, consulta externa, hospitalización o atendidos por primera vez, esto produce que los empleados se expongan a condiciones intensas y mantenidas a lo largo de su empleo convirtiéndose en actividades peligrosas que causan trastornos osteomusculares (Zamora Albuja, 2019). El personal que labora en el área de estadística del Hospital General Puyo muestra diferentes factores de riesgo debido a las actividades diarias que inciden a los movimientos que afectan al desarrollo psicomotriz normal por la mala posición ergonómica de manos y muñecas (López García \& López Mayorga, 2013).

Este trabajo tiene como objetivo implementar: a) pausas activas, b) ubicación correcta de periféricos informáticos, c) suspensión de celular en el periodo de recuperación y medir la efectividad de la mejora con el Método Check List OCRA en los trabajadores que presentan molestias relacionadas por la ejecución de tareas repetitivas en el Hospital General Puyo.

\section{Metodología}

\section{Cuestionario Nórdico Estandarizado}

En este estudio se utilizó el Cuestionario Estandarizado Nórdico de Kuorinka, siendo un método muy eficaz para evaluar los trastornos musculoesqueléticos iniciales de las extremidades superiores en posiciones similares (Martínez \& Alvarado Muñoz, 2017). Esta herramienta utiliza un cuestionario de 11 preguntas que se aplicaron a una población total de 20 trabajadores del área de estadística del Hospital Puyo bajo criterios de selección que toman en cuenta la antigüedad y presencia en el lugar de trabajo.

El Cuestionario Estandarizado Nórdico de Kuorinka es utilizado para detectar y analizar síntomas musculoesqueléticos en diferentes áreas del cuerpo, en nuestro estudio nos enfocaremos en mano y muñeca producido en el último año previo el cual permite evaluar el nivel de riesgo de intervención temprana (Espín Allán, 2020). Hay 11 preguntas de opción múltiple que recopilan información sobre el dolor, el malestar y la incomodidad (Muñoz, 2021). (Ver Tabla 1). Estas preguntas abordan la presencia de malestar en términos de duración, frecuencia y tratamiento de la mano y muñeca, y las consecuencias debido al cambio de puesto o ausencia laboral. Estos datos se recopilan y se utilizan para determinar la prevalencia de trastornos musculoesqueléticos que causan fatiga laboral (Muñoz, 2021).

Mediante una capacitación y la aplicación del cuestionario los 20 trabajadores conocieron los riesgos ergonómicos que generan la realización de sus actividades, así como las molestias y los trastornos musculoesqueléticos que pueden desarrollar.

\section{Método Check List Ocra}

La evaluación de movimientos repetitivos se puede evaluar de forma cuali-cuantitativa siendo la más precisa el Check List OCRA, $\left(\right.$ Tabla ${ }^{\circ}{ }^{\circ}$ 2) (Palomino-Baldeón, Andia-Paz, CárdenasTerry, Salazar-Abad, \& Ygreda-Mejía, 2019) que permite la prevención de trastornos osteomusculares como la tendinitis del hombro, tenosinovitis de Quervain y el síndrome del 
túnel carpiano (Bulla Mondragón, Marroquín Cruz, \& Hernández Peláez, 2021). El método mide el riesgo en función de la probabilidad de desarrollar trastornos musculoesqueléticos durante un período de tiempo determinado y se centran en la evaluación del riesgo de las extremidades superiores, considerando los siguientes factores de riesgo: repetición, postura, esfuerzo, coacción la falta tiempo para descansar y recuperación, evaluarlos en la vida laboral del trabajador (Palomino-Baldeón et al., 2019).

Los factores de riesgo se califican mediante una escala del 1 al 10, pero otros factores de riesgo pueden alcanzar valores más altos en el Índice Check List OCRA y los riesgos se pueden clasificar de la siguiente manera: optimo, aceptable, muy ligero, ligero, medio o alto (Agredo Gómez \& Arroyo Baena, 2020).

Luego, se proponen acciones correctivas, como mejorar el puesto de trabajo, solicitar vigilancia médica o capacitar específicamente a los trabajadores para obtener el puesto (Vega, 2019).

\section{Resultados}

\section{Cuestionario Nórdico Estandarizado}

Los resultados obtenidos mediante la aplicación del Cuestionario Nórdico muestran que el 65\% de los trabajadores del área de estadística sufren molestias musculoesqueléticas en el último año, el $100 \%$ no necesita reposicionarse por lo que continúan trabajando en dicha área de estadística, el 100\% de los trabajadores no habían sido atendidos por su médico (Tabla N $\circ 1)$.

\section{Tabla $\mathbf{N}^{\circ} 1$.}

Cuestionario Nórdico

\begin{tabular}{ccccc}
\hline $\begin{array}{c}\text { Presenta molestias en } \\
\text { mano y muñeca }\end{array}$ & $\begin{array}{c}\text { Necesitó cambio } \\
\text { de } \\
\text { Puesto }\end{array}$ & $\begin{array}{c}\text { Presentó } \\
\text { molestias en } \\
\text { el último año }\end{array}$ & $\begin{array}{c}\text { Recibió } \\
\text { tratamiento }\end{array}$ \\
\hline $\mathrm{Si}$ & $65 \%$ & $0 \%$ & $65 \%$ & $0 \%$ \\
$\mathrm{No}$ & $35 \%$ & $100 \%$ & $35 \%$ & $100 \%$ \\
\hline
\end{tabular}

Nota: Los resultados consideran condiciones extremas de carga de trabajo (Martínez \& Alvarado Muñoz, 2017).

La incomodidad musculoesquelética ocurre en el $85 \%$ de los trabajadores diestros que usan mouse y teclado y en el $15 \%$ de los trabajadores zurdos. (Tabla $\mathrm{N}^{\circ} 2$ )

Tabla $\mathbf{N}^{\circ} 2$.

Cuestionario Nórdico - Parte afectada

\begin{tabular}{cc}
\hline Parte afectada & Porcentaje \\
\hline Derecha & $85 \%$ \\
Izquierda & $15 \%$ \\
Ambos & $0 \%$ \\
\hline
\end{tabular}

Nota: Los resultados no consideran réplicas del cuestionario. 
El 90\% de los trabajadores del área de estadística cree que los movimientos repetitivos al utilizar el teclado y el mouse son la causa de su malestar. (Tabla $\mathrm{N}^{\circ} 3$ )

Tabla $\mathbf{N}^{\circ} 3$.

Cuestionario Nórdico - Causa de las molestias.

\begin{tabular}{lc}
\hline Causa de las molestias & Porcentaje \\
\hline Repetitividad de movimientos & $90 \%$ \\
Postura forzada & $10 \%$ \\
\hline
\end{tabular}

Nota: Los resultados consideran condiciones extremas de carga de trabajo, y ambos géneros (Estrada Uribe, 2015).

\section{Método Check List OCRA}

Al aplicar el método Check List OCRA, encontramos que el puesto de estadística mostró un riesgo leve en el lado derecho y un riesgo aceptable en el lado izquierdo. (Tabla $\mathrm{N}^{\circ} 4$ )

Tabla $\mathrm{N}^{\circ} 4$

Método Check List OCRA

\begin{tabular}{|c|c|c|c|c|}
\hline Puesto de trabajo & \multicolumn{2}{|c|}{ Primera evaluación } & \multicolumn{2}{|c|}{ Segunda evaluación } \\
\hline Factores: & $\begin{array}{l}\text { Lado } \\
\text { derecho }\end{array}$ & $\begin{array}{l}\text { Lado } \\
\text { izquierdo }\end{array}$ & $\begin{array}{l}\text { Lado } \\
\text { derecho }\end{array}$ & $\begin{array}{l}\text { Lado } \\
\text { izquierdo }\end{array}$ \\
\hline $\begin{array}{ll}\text { Factor } & \mathrm{de} \\
\text { recuperación } & \end{array}$ & 3 & 2 & 2 & 2 \\
\hline $\begin{array}{l}\text { Factor de } \\
\text { frecuencia }\end{array}$ & 2.5 & 2 & 2 & 1.5 \\
\hline Factor de fuerza & 0 & 0 & 0 & 0 \\
\hline Factor de postura & 4 & 3 & 2 & 2 \\
\hline $\begin{array}{l}\text { Factores } \\
\text { adicionales }\end{array}$ & 2 & 1 & 1 & 1 \\
\hline Multiplicador & 0.75 & 0.75 & 0.75 & 0.75 \\
\hline $\begin{array}{l}\text { Valor Check List } \\
\text { OCRA }\end{array}$ & 8.62 & 5.25 & 5.25 & 4,87 \\
\hline Nivel de riesgo & $\begin{array}{l}\text { Muy } \\
\text { ligero }\end{array}$ & Aceptable & $\begin{array}{l}\text { Muy } \\
\text { ligero }\end{array}$ & Óptimo \\
\hline
\end{tabular}

Nota: Los resultados consideran la implementación de las medidas correctivas en las mismas condiciones (Piñol, Altet, \& Roldán, 2018).

\section{Discusión}

Los resultados de este trabajo son similares al estudio realizado en Ecuador por la autora Mirell Gutiérrez (2015), en su artículo "Sintomatología músculo esquelética de miembros superiores en personal expuesto a movimientos repetitivos y su relación con el tiempo de exposición en una empresa de manufactura y su plan de control ", donde encontró la presencia de molestias musculo esquelética en los miembros superiores de 
una empresa de manufactura con la utilización prolongada de teclado y mouse los cuales se relacionaban con movimientos repetitivos que fue el factor de riesgo más importante, seguido de posturas forzadas que resultan en un nivel intolerable de riesgo en su puesto de trabajo (Gutiérrez Soriano, 2015).

De igual manera, los resultados son consistentes con otros estudios, en este caso la autora

Para contrastar estos resultados se debería considerar el uso de biomarcadores en la identificación de trastornos musculoesqueléticos a través de la evaluación de las extremidades superiores utilizando herramientas de diagnóstico como la electromiografía (Rodríguez-García, Dorantes-Méndez, \& Mendoza Gutiérrez, 2017).

\section{Conclusiones}

- Los resultados obtenidos del factor postural tuvieron un valor de 4 que están directamente relacionados con el impacto del $65 \%$ del personal del área de estadística del Hospital General Puyo debido al trabajo rotativo con una duración de 8 horas diarias de exposición, según el Check List OCRA y el Cuestionario Nórdico. Por otro lado, con la evaluación inicial del Índice OCRA se encontró un valor de 8,62 en el lado derecho que corresponde a un riesgo leve y en el lado izquierdo 5,25 que determina un riesgo aceptable.

- Para mejorar el diseño del lugar, se debe considerar el control de las variables que determinaron valores ligeros en la investigación como: factor postural, repetibilidad de movimiento al usar periféricos informáticos y mejora del tiempo de pausas activas.

- Luego de aplicar estos métodos, se logró disminuir un 80\% de la puntuación del Check List OCRA con valores de 5,25 para el lado derecho y un riesgo aceptable, además un valor de 4,87 y un riesgo ergonómico óptimo para el lado izquierdo, por lo que se determinó que existe un riesgo leve para el lado derecho y aceptable en el izquierdo por lo que es necesario realizar acciones permanentes en este puesto de trabajo, considerando: a) el entrenamiento y adiestramiento en posiciones y movimientos correctos para el cumplimiento de las actividades laborales, b) establecer puntos de descanso centrados en la mano y la muñeca que permitan la recuperación de la fatiga muscular, movimientos relacionados con el trabajo reducen o controlan el riesgo laboral al que están expuestos.

\section{Referencias bibliográficas}

Agredo Gómez, L. F., \& Arroyo Baena, M. A. (2020). Guía para la clasificación del origen del síndrome de manguito rotador utilizando el método Ocra. Universidad Santiago de Cali,

Angamarca Curipoma, L. P., \& Changoluisa Changoluisa, G. M. (2020). Trastornos osteomusculares en miembros superiores por movimientos repetitivos en personas que laboran en el área administrativa con pantallas de visualización de datos en edades comprendidas entre 23 a 50 años en el Distrito de Salud 17D07 y la 
propuesta de medidas correctoras en el período septiembre 2019-febrero 2020. Quito: UCE,

Armesto, I. G., \& Piñeiro, R. G. 5.3. DOLOR DE MUÑECA Y MANO. ABORDAJE MÉDICO DEL DOLOR EN, 73.

Bulla Mondragón, L. M., Marroquín Cruz, L. Y., \& Hernández Peláez, S. D. (2021). Estudio de los trastornos osteomusculares mediante el método OCRA check list y su incidencia en las alteraciones musculares en los trabajadores de una plaza de mercado de Cundinamarca, Colombia. Corporación Universitaria Minuto de Dios,

Chacín, A. J. P., González, A. I., \& Peñaloza, D. W. (2020). Educación superior e investigación en Latinoamérica: Transición al uso de tecnologías digitales por Covid-19. Revista de Ciencias Sociales, 26(3), 98-117.

Cisneros-Prieto, M. A., \& Cisneros-Rodríguez, Y. (2015). Los accidentes laborales, su impacto económico y social. Ciencias Holguín, 21(3), 1-11.

Cordoba Perez, D. (2018). Prevalencia de desórdenes musculoesqueléticos en trabajadores de oficina y factores relacionados: revisión de la literatura. Universidad del Rosario,

Espín Allán, L. M. (2020). Validación del cuestionario nórdico para la identificación de molestias osteomusculares, y la comparación con la valoración médica, en población trabajadora de plantaciones florícolas.

Espinoza Huaman, J. E. (2018). Estudio de factores de riesgo ergonómico físico en el personal administrativo de la construcción del Hospital Daniel Alcides CarriónPasco.

Gómez C, Á. G., Fernández A, Castro F, Vega V, Rodríguez R, et al. (2017). La investigación científica y las formas de titulación. Aspectos conceptuales y prácticos. (Q. E. J. d. Ecuador; Ed.).

Gutiérrez Soriano, M. (2015). Sintomatología músculo esquelética de miembros superiores en personal expuesto a movimientos repetitivos y su relación con el tiempo de exposición en una empresa de manufactura y su plan de control. Universidad Internacional SEK,

López García, A. M., \& López Mayorga, M. M. (2013). Síntomas y riesgos musculoesqueléticos, en trabajadores de ofcina del Hospital César Amador de Matagalpa y Mauricio Abdalah de Chinandega.

Martínez, M., \& Alvarado Muñoz, R. (2017). Validación del Cuestionario Nórdico Estandarizado de Síntomas Musculoesqueléticos para la población trabajadora chilena, adicionando una escala de dolor. 
Moller, S. (2020). Intervenciones sociosanitarias y uso de las tecnologías de la industria 4.0 para enfrentar la enfermedad por coronavirus (COVID-19) en América Latina y el Caribe.

Muñoz, E. L. G. (2021). Estudio de validez y confiabilidad del cuestionario nórdico estandarizado, para detección de síntomas musculoesqueléticos en población mexicana. Ergonomía, Investigación y Desarrollo, 3(1), 8-17.

Palomino-Baldeón, J. C., Andia-Paz, G., Cárdenas-Terry, M., Salazar-Abad, J. K., \& Ygreda-Mejía, P. (2019). Intervención ergonómica evaluada por Ocra Check List a digitadores, Lima-2015. Revista de la Asociación Española de Especialistas en Medicina del Trabajo, 28(3), 195-203.

Pineda Rengel, C. M. (2019). Síndrome de quervain en adultos, diagnostico, tratamiento y control.

Rengifo Villavicencio, A. (2020). Síntomas musculoesqueleticos en el personal asistencial del CAP III Iquitos Essalud, utilizando el cuestionario nordico de kuorinka estandarizado.

Rodríguez-García, M., Dorantes-Méndez, G., \& Mendoza Gutiérrez, M. (2017). Desarrollo de una prótesis para desarticulado de muñeca controlada por señales de electromiografía. Revista mexicana de ingeniería biomédica, 38(3), 602-620.

Sagbay, C. A. U. (2021). Estudio comparativo entre el método Check List OCRA y RULA-RULER para la evaluación de riesgos ergonómicos asociados con sufrir enfermedades musculoesqueléticas en operativos de línea. UNIVERSIDAD DE CUENCA,

Salas Guerra, D. T., \& Díaz López, L. R. (2017). Factores de riesgo asociados a alteraciones osteomusculares de la muñeca en trabajadores del área administrativa de una entidad promotora de salud del departamento de córdoba durante el año 2016.

Uribe, N. C. (2019). Estudio ergonómico del proceso de armado y encolado de paneles. Ergonomía, Investigación y Desarrollo, 1(3), 138-157.

Vega, S. (2019). Instituto Nacional de seguridad e higiene en el trabajo. Recuperado el, 15.

Zamora Albuja, C. A. (2019). CONSTRUCCIÓN DE UN ELEMENTO PROGRAMADOR PARA LA CONFIGURACIÓN DE DISPOSITIVOS NFC PASIVOS, PARA PERMITIR LA VISUALIZACIÓN DE FICHAS MÉDICAS DE PACIENTES EN UN SMARTPHONE. Quito, 


\section{PARA CITAR EL ARTÍCULO INDEXADO.}

Freire Ramos, E. del R., Córdova Suarez, M. A., Villacres Cevallos, E. P., \& Cuenca Cajamarca, M. F. (2021). Prevención de Movimientos Repetitivos y Tenosinovitis de Quervain en el Área de Estadística del Hospital General Puyo. Anatomía Digital, 4(3.1), 18-27. https://doi.org/10.33262/anatomiadigital.v4i3.1.1851

\section{Liencia}

El artículo que se publica es de exclusiva responsabilidad de los autores y no necesariamente reflejan el pensamiento de la Revista Anatomía Digital.

El artículo queda en propiedad de la revista y, por tanto, su publicación parcial y/o total en otro medio tiene que ser autorizado por el director de la Revista Anatomía Digital.
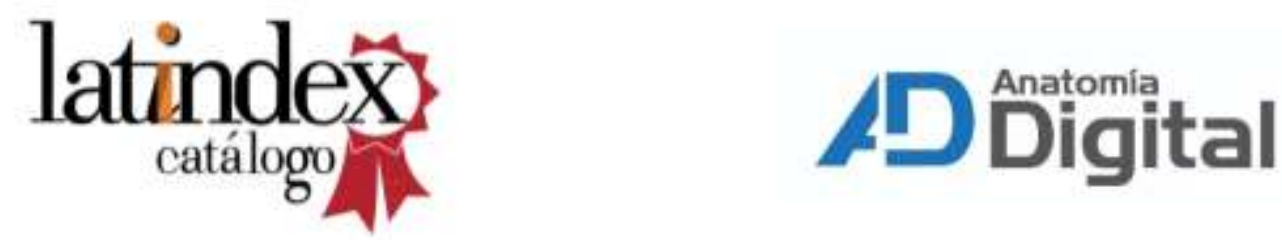